\title{
COMPUTER SIMULATION OF FUNCTIONALIZED CARBON NANOTUBES AND GRAPHENE
}

\author{
Alexey V. Kosobutsky a,*, Sergey D. Shandakov a, Albert G. Nasibulin b \\ ${ }^{a}$ Kemerovo State University, \\ Krasnaya Str. 6, Kemerovo, 650043 Russian Federation \\ ${ }^{\mathrm{b}}$ Skolkovo Institute of Science and Technology, \\ Nobel Str. 3, Moscow, 143005 Russian Federation \\ * e-mail: kosobutsky@kemsu.ru
}

Received September 28, 2016;

Accepted in revised form November 15, 2016;

Published December 30, 2016

\begin{abstract}
Nanomaterials based on carbon nanotubes (CNT) and graphenes attract a lot of attention of researchers as the materials capable to raise the development of various industries to the new level, and first of all, of the chemical and electronic sectors. In addition to known experimental methods, new nanosystems are widely studied using advanced tools of quantum-chemical approaches. Modern theoretical methods are of great interest due to their ability to interpret known experimental facts and predict properties of non-synthesized compounds yet. This paper reviews results of theoretical studies performed using the density functional theory (DFT) methods to obtain data on the structure and electronic properties of single-walled CNT and graphene, modified with various impurities, with covalent-ionic and non-covalent binding mechanisms. New computational methods are briefly described that are currently employed to treat the dispersion interaction and enhance possibilities of DFT tools in systems where the van der Waals forces play a significant role. Particular attention is paid to the characteristics of carbon nanomaterials containing technologically important hydroxyl, carboxyl and amino groups. It is shown that the specific peculiarity of band structures of discussed in the literature CNT functionalized by $\mathrm{OH}, \mathrm{COOH}, \mathrm{NHn}$ and $\mathrm{CONH} 2$ groups is the partially occupied band in the neighborhood of the Fermi level, which directly affects the CNT conductivity. Modification of graphene layers is analyzed that interact with hydrogen, fluorine, bases of nucleic acids and the metal substrate surface. We also provide accuracy estimates for the calculations of interatomic bond lengths, interaction energy and band gap carried out in the literature using a variety of DFT approximations.
\end{abstract}

Keywords: carbon nanotubes, graphene, functionalization, density functional theory

DOI: $10.21603 / 2500-1418-2016-1-2-114-125$.

\section{INTRODUCTION}

Experimental study of the properties of individual carbon nanotubes (CNT) and graphene layers of atomic thickness is still the challenge due to high requirements to the equipment and time-consuming manipulation of nanoscale objects. With the rapid development of computer technologies for the last 20 years, theoretic methods to study nanosystems are widely acknowledged. Theoretical modeling allows the tracing of the "structure - property" relations at full control of the composition and structure of test objects which is often impossible for experimental methods. Such studies are particularly important when characterizing functionalized CNT and graphenes, and the most important is the level of accuracy to be suggested by the particular approach.

The density functional theory (DFT) that is based on the Kohn-Sham formalism [1] is widely recognized when addressing a wide range of physical and chemical problems. DFT methods that were initially used in the field of solid state physics, with the development of new computational algorithms, have become one of the most employed to model molecular systems, clusters and nanomaterials. Two factors largely contributed to widely use the DFT as follow: lesser demand of computational resources as compared to traditional non-empirical quantum-chemical approaches (Hartree-Fock-Roothan theory, configuration interaction, coupled clusters, etc.), with very high computational costs as well as the development of effective exchangecorrelation functionals, optimized for different tasks $[1,2]$.

Modern calculations within DFT show a wide variety of implemented approaches based on pseudopotential methods (ab initio norm-conserving or ultrasoft pseudopotentials with using the plane wave (PP-PW) or localized orbital basis sets), linear muffin-tin orbitals (LMTO), linearized augmented plane wave (LAPW). Today, the PP-PW approach is the typical method to calculate energetic characteristics of periodic systems which is able to ensure the accuracy close to attainable by such full-potential methods as FP-LAPW for many applications, and is simpler in practice. It is seen in practice that approximations introduced when describing the exchange-correlation interaction have the decisive role for the numerical simulation of

Please cite this article in press as: Kosobutsky A.V., Shandakov S.D., Nasibulin A.G. Computer simulation of functionalized carbon nanotubes and graphene. Science Evolution, 2016, vol. 1, no. 2, pp. 114-125. doi: 10.21603/2500-1418-2016-1-2-114-125.

Copyright (C) 2016, KemSU. This is an open access article distributed under the terms of the Creative Commons Attribution 4.0 International License (http:// creativecommons.org/licenses/by/4.0/), allowing third parties to copy and redistribute the material in any medium or format and to remix, transform, and build upon the material for any purpose, even commercially, provided the original work is properly cited and states its license. This article is published with open access at http:// science-evolution.ru/ 
electronic properties rather than the specific calculation scheme to solve the Kohn-Sham equations. In modern works, the local density approximation (LDA) or the generalized gradient approximation (GGA) is normally used.Calculations made within DFT-LDA/GGA show the high accuracy in describing the structural parameters of metal and ionic-covalent systems (discrepancy with experiment at the level of a few percent) at normal conditions and under pressure, electron energy spectra in the region of occupied states and vibrational properties. The class of hybrid functionals (B3LYP, PBE0, HSE06), containing the certain amount of Hartree-Fock exchange allows to increase the predicting accuracy of electronic spectrum computations, in particular, of interband transitions and band gap values.

The applicability of the theory to describe the longrange dispersion forces is significant to describe structural and energetic characteristics of CNT bundles, and of adsorbed molecules on the CNT and graphene surface. Widely used LDA and GGA functionals demonstrate low productivity when solving tasks of such class. However, in recent years significant steps have been made in this direction owing to the efforts of international research groups, which allow developing a number of methods to treat dispersion interaction that may be classified based on the level of accuracy provided. Due to its significance in modeling functionalized carbon nanomaterials, such techniques deserve to be discussed separately. The work [3] considers the hierarchy classification where stair climbing up means the transition to more advanced and sophisticated approaches. The DCACP method is taken as the basis of the hierarchy ladder that is conditionally referred to the zero level. The calculations within the DCACP are made using the atom centered modified potentials. This approach may be of interest to calculate complex compounds, but its disadvantage is the need for careful potential adjustment for each element and implementation difficulties on the full-potential level. Computational schemes which are arranged on the first level ensure asymptotics $-1 / r^{6}$ for the dipole-dipole interaction by introduction of relevant semiempirical coefficients $C_{6}$. However, the most promising are methods that do not rely on external inputs, but determine the dispersion interaction energy directly as the electron density function. This is primarily the more general strategy, and thus, such methods are on the third level of the hierarchy [3]. The basis of these methods is the use of recently developed long-range non-local correlation functionals as the additional term in the expression for total exchangecorrelation energy.

\section{MATERIALS AND METHODS}

Below we briefly describe the main computational methods currently used to treat the dispersion interaction when modeling the adsorption on the surface of the CNT and graphene. The basic requirement for any calculation scheme based on DFT is the provision of asymptotic behavior of interaction energy according to the law $-1 / r^{6}$ for the particle interaction in the gas phase, where $r$ is a distance between particles. The simplest approach to achieve this goal is the introduction of an additional term in the total energy expression that will include all missing long-range interactions:

$$
E_{\mathrm{tot}}=E_{\mathrm{DFT}}+E_{\mathrm{disp}} .
$$

In the formula (1) $E_{\mathrm{DFT}}$ is the DFT energy calculated using the relevant exchange-correlation functional, $E_{\text {disp }}-$ term which includes the dispersion effects:

$$
E_{\mathrm{disp}}=-\sum_{A, B} C_{6}^{A, B} / r_{A, B}^{6}
$$

Dispersion coefficient $C_{6}^{A, B}$ depends on the type of particles $A$ and $B$. Due to divergence of (2) at $r \rightarrow 0$, the expression (2) is practically used with the function $f$ $\left(r_{A B}, A, B\right)$ rapidly damped at smaller distances:

$$
E_{\mathrm{disp}}=-\sum_{A, B} f\left(r_{A B}, A, B\right) C_{6}^{A, B} / r_{A, B}^{6} .
$$

The methods on the first step of the classification scheme use isotropic (i.e., not direction-dependent) and constant coefficients; these methods are commonly known as DFT-D (Fig. 1) in the literature. In 2006, S. Grimm published the computational scheme identified as DFT-D2 that calculated dispersion coefficient based on the formula that includes ionization potentials and static polarizabilities of isolated atoms [4]. The data for all elements up to $\mathrm{Xe}$ is available to ensure high interest in the work [4], and currently, this is probably the most widely used approach to treat dispersion effects.

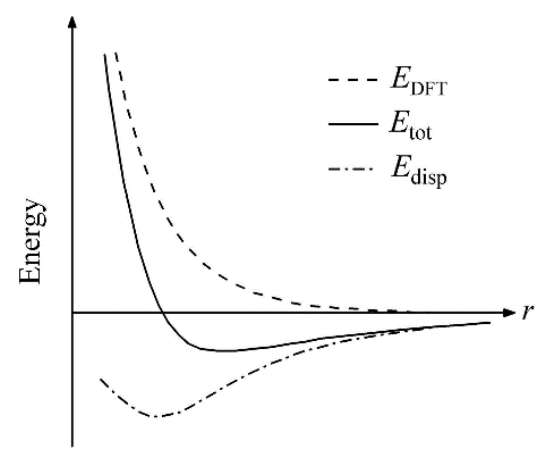

Fig. 1. Schematic dependence of the binding energy $E_{\text {tot }}$ on the distance $r$ between particles when calculating within the DFT-D method and separate contributions to the value $E_{\mathrm{tot}}=E_{\mathrm{DFT}}+E_{\mathrm{disp}}$.

In 2009, Tkatchenko and Scheffler [5] proposed an approach that relies on atomic polarizability and atomic coefficients $C_{6}$ to calculate the dispersion energy. These quantities are sufficient to obtain the pair coefficients $C_{6}$. The effective atomic volumes are used to treat the dependence on the environment. When calculating the considered system's properties, the electron density of molecule is divided into the densities of individual atoms (as per Hirshfield scheme) and the contribution of each atom is compared to the density of free atoms. This ratio is then used to scale the $C_{6}$ coefficient of a reference atom that specifies the value of dispersion energy. The accuracy of the scheme of Tkatchenko and Scheffler to quantify $C_{6}$ is quite high with error estimate of about 5.4\% (MAPD). 
Methods described above require pre-defined input parameters to evaluate the dispersion interaction, whether $C_{6}$ factors are derived directly or from atomic polarizabilities. Of great concern are approaches that do not rely on external inputs but express the energy of dispersion interaction directly through the electron density. The basis of these methods is the use of recently developed non-local correlation functional of the form:

$$
E_{c}^{n l}=\iint d r d r^{\prime} n(r) \varphi\left(r, r^{\prime}\right) n\left(r^{\prime}\right),
$$

where $\varphi\left(r, r^{\prime}\right)$ - particular function depending on the coordinate difference $\left|r-r^{\prime}\right|$, electron density $n(r)$ and its gradient. In 2004, M. Dion et al [6] suggested the equation for $\varphi\left(r, r^{\prime}\right)$ that is appropriate for systems with arbitrary geometry and ensures the asymptotic behavior as required $O\left(-1 /\left|r-r^{\prime}\right|^{6}\right)$. Within this approach, the full exchange-correlation energy is defined by the expression:

$$
E_{X C}=E_{x}^{G G A}+E_{c}^{L D A}+E_{c}^{n l},
$$

where the terms on the right side are, respectively, the exchange energy in revPBE approximation, LDA correlation energy and non-local correlation energy term. Functionals of this type are called the van der Waals density functionals (vdW-DF). The calculation method is named accordingly that uses functionals of that type.

It should be noted that the vdW-DF method is the significant theoretical development, since within this approach, the description of the dispersion interaction is directly included in the expression for the DFT total energy where the correlations for all distance ranges are written in the single formula. The introduction of the original vdW-DF scheme was followed by a number of works to analyze and raise the effectiveness of this method. To date, a set of modified functionals is offerred such as vdW-DF2 [7], vdW$\mathrm{DF}\left(\mathrm{C} 09_{\mathrm{x}}\right)$ [8], rev-vdW-DF2 [9] and etc. Self-consisted versions of these methods are implemented in widely used computation packages such as VASP, SIESTA, Quantum ESPRESSO and QChem.

The analysis of the published theoretical results shows a significant increase in the accuracy of calculations of structural parameters and energetic characteristics of layered and molecular crystals [10,11], as well as nanostructured materials using functionals of $\mathrm{vdW}-\mathrm{DF}$ family. In general, the systems with the number of atoms to $\sim 1000$ (in the periodicity element, in case of using periodic boundary conditions) are currently available to model within DFT using standard computing clusters. For systems with larger number of atoms $\left(10^{4}-10^{5}\right)$, the application of semi-empirical schemes is reasonable. In addition, the combined approach is of interest, when DFT is used only to simulate critical parts of nanostructures, while the remaining parts are described more roughly. However, this method requires the more profound system analysis to isolate its key fragments and the resulting values are not always accurate. The work [12] can be cited as an example where the accuracy of various nanotube modeling methods was estimated on the example of metallic CNT with the chirality indices $(5,5)$ with 2-phenylimidazolines attached. The authors considered $a b$ initio method (calculations within the density functional theory using the Gaussian basic functions of 3-21G, 6-31G sets and B3LYP hybrid exchange-correlation functional) and the combined ONIOM scheme separating the polyatomic system of interest into the smaller part with properties to be defined with the accurate $a b$ initio approach and the largest part of the rest of atoms to be considered semiempirically. It is concluded by the results comparison of the binding energy calculation that the full DFT method is preferable when choosing the way for nanotube simulation, while the simplified ONIOM approach can give good accuracy results providing properties of sufficiently large number of atoms are treated $a b$ initio. Therefore, the present review mainly considers works performed within the first approach, that is, using a common calculation procedure for all structural elements.

The impact of various molecules attached to singlewalled CNT (SWCNT) on their electron properties was studied from first principles in works [13-21]. Hydroxyl and carboxyl groups are thought to be one of the most important impurities; these groups actively bind to CNT surface during the standard treatment by acids. Moreover, the introduction of carboxyl group $(-\mathrm{COOH})$ is of interest due to the possibility of further replacement of $-\mathrm{OH}$ as the group component by more complicated molecular structures (for example, amino acids, DNA, amino groups) using standard chemical reactions [13]. To study the impact of doping by -OH groups in [14], evaluations were performed in terms of total energy, band structure and state density of zigzag semiconducting SWCNT (10, 0) with $\mathrm{OH}$ molecules attached to walls thereof. Calculations were performed within DFT using the Vanderbilt ultrasoft pseudopotentials and the plane-wave basis and exchange-correlation effects were described within PW92-GGA. To simulate the nanotube properties, the periodic boundary conditions and the supercell approximation were used. The calculations proved the stability of CNT-OH complex and gave the value of the bond energy for hydroxyl group $E_{\mathrm{b}}(\mathrm{OH})=0.78 \mathrm{eV}$, where the angle between $\mathrm{C}-\mathrm{O}$ and $\mathrm{C}-\mathrm{C}$ bonds of the optimized structure was $\sim 100^{\circ}$, and the $\mathrm{C}-\mathrm{O}$ bond length was equal to $1.48 \AA$. It should be noted that the length of neighboring $\mathrm{C}-\mathrm{C}$ bonds estimated at $1.52 \AA$ is greater than that in the initial free CNT (1.42 $\AA)$. It tells on the local distortion of its structure. It is shown that the attachment of $\mathrm{OH}$ group to the CNT wall results in the additional peak in the density of states near the Fermi level. In [15], the hydroxyl groups doping was studied based on three CNT varieties with similar diameters (Fig. 2 ), while the $\mathrm{OH}$ groups joined to open ends of nanotubes. Resulting from the multiple attachment of $\mathrm{OH}$ groups, strong hydrogen bond formation is reported that stabilize the structure of doped CNT in addition. Selectivity of hydroxylation process is the interesting finding. Energy consumption calculations show that hydroxylation by replacement of $\mathrm{H}$ atoms on CNT open ends is the endothermic process that is energetically more beneficial for armchair-like and chiral CNT as compared with zigzag tubes. 

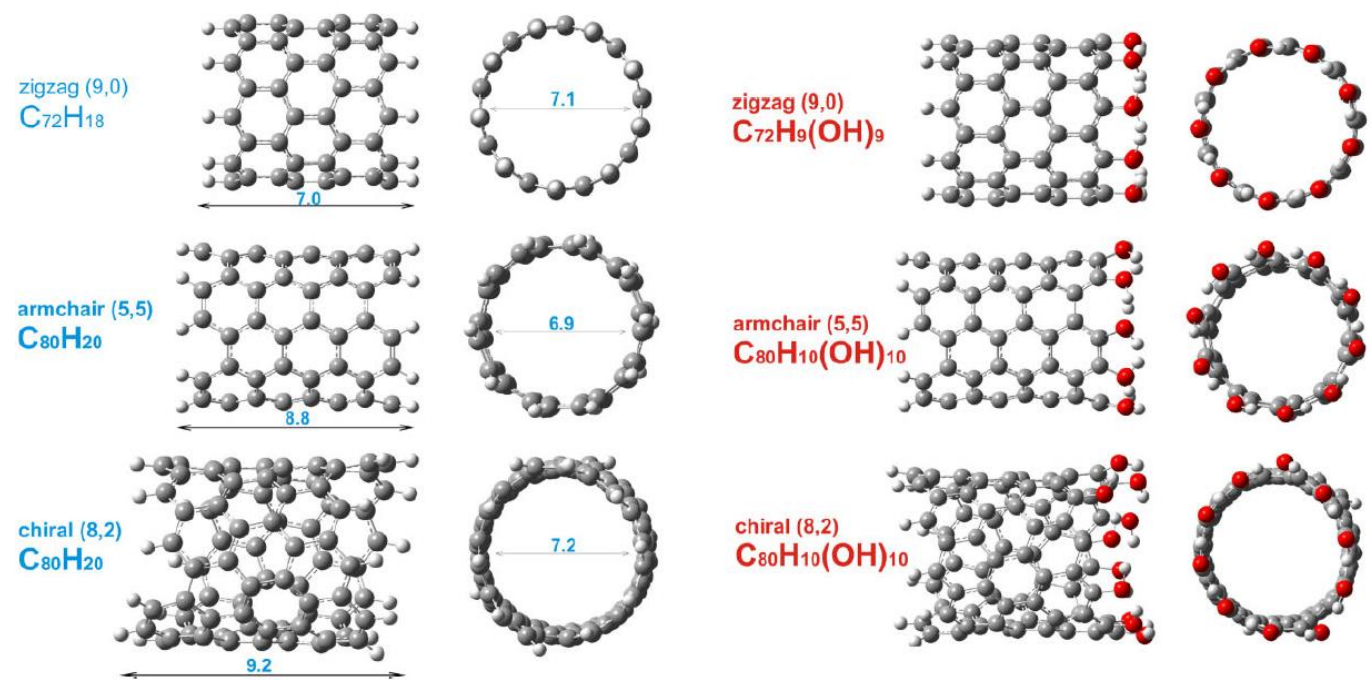

Fig. 2. Optimized structures of three CNT types with different chirality indices before and after doping by OH groups as per [15].

In $[16,17]$ the first-principles studies of SWCNT functionalized by covalent bonding with carboxyl group $(-\mathrm{COOH})$ and groups containing the nitrogen atom $\left(-\mathrm{NH}_{2}\right.$ and $\left.-\mathrm{CONH}_{2}\right)$ were performed. As in the case of -OH attachment, the geometry distortion of the tube wall in the neighbourhoods of impurities was identified that may be explained by the local change in the hybridization type of atomic orbitals $s p^{2} \rightarrow s p^{3}$ due to impurity introduction and additional covalent $\mathrm{C}-\mathrm{C}$ and C-N bond formation. According to calculations [16], the largest binding energy $E_{\mathrm{b}}$ to the $\operatorname{SWCNT}(8,0)$ surface has the amino group $(1.88 \mathrm{eV})$, and the least - amide radical $(1.22 \mathrm{eV})$, whereas $E_{\mathrm{b}}(\mathrm{COOH})=1.58 \mathrm{eV}$. It is worth noting that the given estimates should be considered with caution since energetic characteristics in [16] were evaluated using the localized basis and thus, they are subjected to the well-known influence of the so-called basis set superposition error (BSSE). The consistent BSSE treatment always results in the decrease in absolute value of the binding energy, whereas correction values can be up to tens of percent of initial quantities. Perhaps, the considerable portion of difference between calculated binding energies of $\mathrm{COOH}$ group in [16] and [18] is explained by this factor. The latter work compared the energetic characteristics of carboxyl-functionalized SWCNT $(10,0)$ with the ideal initial geometry of the surface and with the Stone-Wales structural defects. A Stone-Wales defect is the typical topological defect of nanotubes and consists of two pairs of five- and seven-membered rings (Fig. 3 ). It is found based on all-electron calculations of the total energy in [18] that the $\mathrm{COOH}$ bonding energy to various defective sites of CNT $(1.26-2.00 \mathrm{eV})$ is considerably higher than that with the ideal CNT $(0.92 \mathrm{eV})$. The most favorable position of $\mathrm{COOH}$ group is found connected with one of defect atoms where it is shown that the attachment of two $\mathrm{COOH}$ molecules to two central atoms of the Stone-Wales defect results to a more energetically stable structure. It is concluded based on calculations of the CNT-COOH band spectrum on the appearance of partially occupied band with significant dispersion in the energy gap region. The authors suggest that this can allow considerable increase in electrical conductivity of semiconducting CNT. Similar results are obtained in [19] with the theoretical modeling of $\mathrm{CNT}(8,0)$ with the Stone-Wales defects functionalized by the amino group.

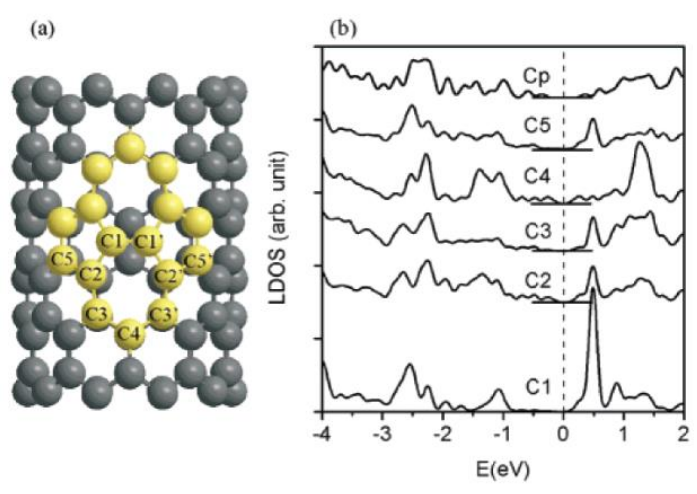

Fig. 3. (a) A wall of zigzag SWCNT $(10,0)$ with the Stone-Wales defect. (b) Contributions to electronic density of state from the numbered carbon atoms [18].

It is important to note that the reconstruction of electronic spectra of semiconducting $\mathrm{CNT}$ resulting from the doping by $\mathrm{OH}, \mathrm{COOH}, \mathrm{NH}_{n}, \mathrm{CONH}_{2}$ impurities does not significantly depend on the type of the group attached. A characteristic feature of band spectra of structures studied in literature is the presence of partially occupied band in the neighborhood of the Fermi level (Fig. 4) which directly affects the CNT conductivity. According to calculations of atomic charges, the said impurities pull a part of electron density of the tube. Thus, acting like the $p$-type impurity, external groups increase the conductivity of individual CNT, which will also facilitate the improvement of electrical characteristics of films based on nanotubes. Besides, the CNT surface decoration with $\mathrm{OH}, \mathrm{COOH}, \mathrm{NH}_{\mathrm{n}}$ and other groups allows a decrease in interaction energy of neighboring nanotubes. Thus, it reduces the tendency of CNT bundle formation and, consequently, facilitates their dispersion in the solution. 

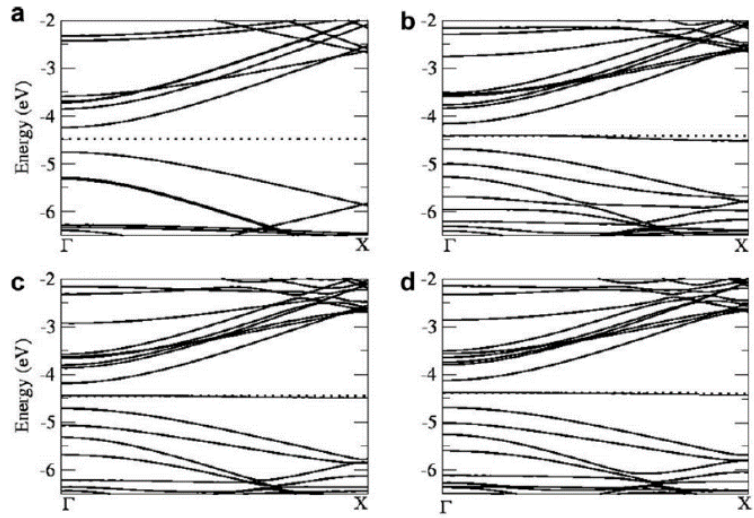

Fig. 4. Electronic band structures of (a) pure SWCNT (8, $0)$, (b) $\operatorname{SWCNT}(8,0)-\mathrm{NH}_{2}$, (c) $\operatorname{SWCNT}(8,0)-\mathrm{COOH}$, (d) SWCNT $(8,0)-\mathrm{CONH}_{2}$ [16]. The dotted line indicates the Fermi level position.

As in [18, 19], the works [20, 21] also addressed the issue of molecule interaction with defective CNT sites. These papers share a common theoretical research method known as the approximate two-layer approach ONIOM. Studies in [20,21] of interaction of the nitrogen molecules $\mathrm{N}_{2}$ and methylamine $\mathrm{CH}_{3} \mathrm{NH}_{2}$ with the CNT walls and open ends showed that the attachment of molecules to carbon atoms at the open ends are energetically more favorable than attachment to the wall. Therefore, the adsorption capacity of CNT with open ends is higher than in case of tubes with closed ends.

The properties of doped graphene have been so far less studied in comparison with CNT-based systems, nevertheless, the number of works in this field is growing rapidly. The important role of modern quantum chemical methods is worth noting in the investigation of graphene structures. Thus, in 2007, based on $a b$ initio calculations [22] the stability of hydrogenated graphene (graphane) was predicted as well as the graphene transition from semimetallic to insulating state, when saturated with hydrogen atoms, which is successfully proved by further experimental works [23]. This effect is of great importance for electronics applications, since it allows control of the

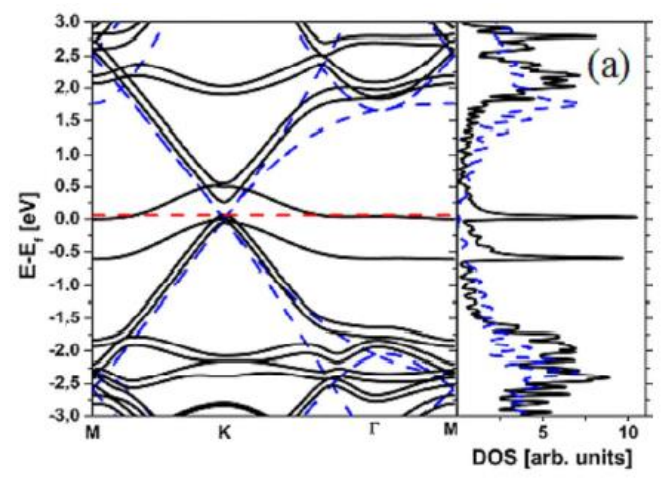

band gap width that affects the concentration of charge carriers. In addition to doping, the graphene conductivity may be also changed by forming vacancies [24, 25], by introducing topological defects [26-28] and by creation of combined systems, for example, based on graphene nano-ribbons and graphane [29]. So, according to the last paper, zigzag hybrid graphane-graphene nano-ribbons give the possibility to control the width of the band gap by changing the length of the graphane fragment and the width of the nanoribbon. The Fig. 5 shows the calculation results of nanoribbon atomic structure sudied in [29].

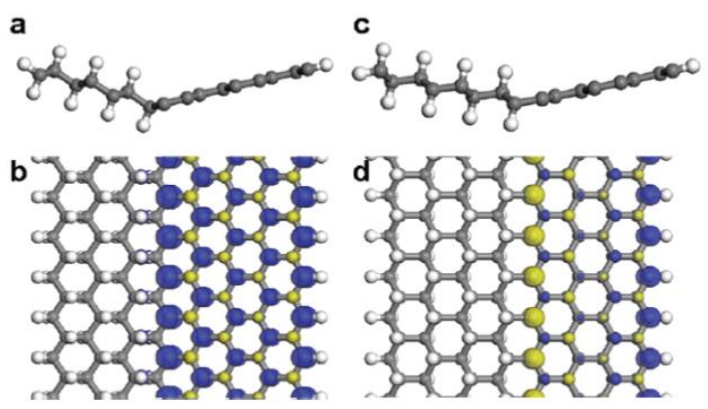

Fig. 5. Optimized structures of hybrid nanoribbons based on graphene and graphane [29] with different covering of the interface zigzag carbon chain with hydrogen atoms. Grey balls $-\mathrm{C}$ atoms, white balls $-\mathrm{H}$ atoms. Colored areas indicate the spin density values: yellow stands for positive values, and the blue color negative value.

The role of impurities $-\mathrm{H},-\mathrm{OH}$ and $-\mathrm{NH}_{2}$ for the graphene was considered in [30-32] based on the single methodology (PBE-GGA approximation for exchangecorrelation functional, norm-conserving pseudopotentials, split-valence basis sets and periodic boundary conditions), using the SIESTA package for quantum chemical simulation. Binding energy of imputiries is estimated, $E_{\mathrm{b}}(\mathrm{OH})=\sim 1.5 \mathrm{eV}, E_{\mathrm{b}}\left(\mathrm{NH}_{2}\right)=\sim 0.8 \mathrm{eV}$ that exhibit quite high stability of functionalized graphene layers. The relevant calculations of electron structure is also performed (Fig. 6).

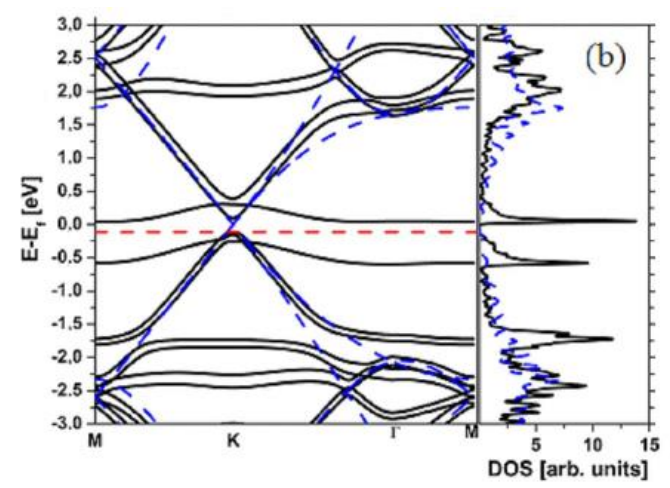

Fig. 6. Electronic band structures and density of states for graphene monolayer functionalized by (a) OH groups, (b) $\mathrm{NH}_{2}$ groups (dark lines) [32]. The blue dotted line shows the band spectrum of the initial pure graphene. The red dotted line indicates the position of HOMO level. 
It is found that at "small" concentration of $\mathrm{OH}$ groups (one $\mathrm{OH}$ group per $18 \mathrm{C}$ atoms), the GR-OH system (where GR stands for graphene) remains semimetallic (Fig. 6(a)), whereas at "higher" concentrations (one $\mathrm{OH}$ group per $8 \mathrm{C}$ atoms), it switches to a semiconducting state. At the same time, doping by $\mathrm{NH}_{2}$ impurities always results in appearance of the band gap in GR-NH $\mathrm{NH}_{2}$ system irrespective of the amino group concentration (Fig. 6(b)). The important thing is that in all cases considered in [32], the band gap is indirect, with minimal transition at $\mathrm{K} \rightarrow \mathrm{M}$. In case of GR-OH, $E_{\mathrm{g}}$ is estimated to be $0.30 \mathrm{eV}$, and for GR-NH$H_{2}$ the $E_{\mathrm{g}}$ value is $0.15 \mathrm{eV}$. It should be noted that evaluations of the band gap width within DFTGGA tend to give significantly underestimated $E_{\mathrm{g}}$ magnitude, which is the known drawback of LDA and GGA approximations in their standard form. Therefore, the values given in [32] should be regarded as values demonstrating a quality trend.

Functionalization of graphene and SWCNT with hydrogen and silyl radicals $\left(-\mathrm{SiH}_{3}\right)$ was studied through the DFT-LDA method in [33]. Chirality indices of studied CNT changed from $(4,4)$ to $(18,0)$, isolated nanotubes were simulated using periodic boundary conditions. The authors have found that the silyl radicals form strong covalent bonds to $\mathrm{CNT}$, and in the energy spectrum of metal tubes (prior to functionalization) the forbidden gap appears, that is, regardless of their chirality they become semiconducting. This effect is associated with enhancement of $s p^{3}$ hybridization during $\mathrm{SiH}_{3}$ joining. This type of functionalization may be useful for reliable CNT fixation on the suitable substrate, which is required for their practical use in various electronic fields. To control the extent of successful attachment of $\mathrm{SiH}_{3}$ groups in practice, the authors [33] propose using the effect of increase of the radial breathing mode $\mathrm{A}_{1 \mathrm{~g}}$ frequency identified by them during functionalization.

A range of researchers also focus on possibilities that give doping by groups containing sulfur [34, 35]. The use of sulfur impurities is regarded as one of ways of self-assembly of nanostructures. Such processes can be realized on surfaces coated with $\mathrm{Au}$ atoms, which is well known to have the high affinity to sulfur. In [36] a theoretical analysis of properties of semiconducting and metallic CNT with - SH groups attached to their walls has been performed. As per calculations, the space between the carbon atom of CNT wall and the sulfur atom is $1.9 \AA$, which is significantly longer than the $\mathrm{C}-\mathrm{O}$ bond length when functionalized by $-\mathrm{OH}$ hydroxyl group. The greater bond length indicates the small SH interaction energy with the CNT wall. In fact, according to [36], in case of semiconducting tubes, $E_{\mathrm{b}}(\mathrm{SH})$ equals to $2-4 \mathrm{kcal} / \mathrm{mol}$, that is, one order less than that for $E_{\mathrm{b}}(\mathrm{OH})$. For metallic CNT, the value of $E_{\mathrm{b}}(\mathrm{SH})$ amounts to $7-9 \mathrm{kcal} / \mathrm{mol}$. It can be concluded from the results of completed researches that the low binding energy of $\mathrm{SH}$ groups extremely impedes obtaining of stable $\mathrm{CNT}-\mathrm{SH}, \mathrm{CNT}-\mathrm{SCH}_{3}$ complexes. It is reasonably supposed in [36] that practical achievements in this field were obtained rather at the expense of structural defects of the tube surface with the known higher reactivity. The intensive mechanical and chemical treatment of samples indirectly indicate the presence of a large number of defects $[34,35]$. An interesting result of [36] is also the conclusion on the chemical inertness of graphene to the - $\mathrm{SH}$ groups.

In [37], CNT functionalization by azomethine ylides was studied. DFT-GGA(PBE) calculation method implemented in the SIESTA and CPMD software packages and first-principles pseudopotentials have been employed. The integration over the Brillouin zone was performed on the Monkhorst-Pack grids containing $4 k$-points. Supercells with CNT inside included 96-144 hydrogen atoms for considered CNT $(4,4),(8,0),(6,6)(9,0)$. The geometry optimization resulted in determination of the interatomic bond lengths and binding energy $E_{\mathrm{b}}$ of azomethine-ylides attached. For various CNT studied, $E_{\mathrm{b}}$ varies within $0.46-1.54 \mathrm{eV}$. It is found that pyrrolidine rings are attached at some angle to CNT axis in case of armchair tubes and to the segment parallel to CNT axis for zigzag tubes. The functionalization of metallic CNT leads to a modification of their electron structure and the band gap appears in the band spectrum. At the same time, the functionalization of initially semiconducting CNT does not result in the substantial change of their band gap width.

In [38], the combined experimental and theoretical approach was employed to study CNT properties upon polyamine treatment. It is found that hydrogenated CNT do not form dense bundles as compared with pure CNT, due to the strong interaction with the solvent molecules (methanol, ethanol, etc.). To study the hydrogenation process, the influence of hydrogen atoms on CNT structure and interaction between single CNT, the ab initio calculation technique within the DFTLDA was used. It is determined that the gain in binding energy as compared with the free $\mathrm{H}_{2}$ molecule is 0.15 $\mathrm{eV}$ when placing $\mathrm{H}$ atoms (isolated orthohydrogen) on the CNT surface which is responsible for the possibility of hydrogen chemisorption found experimentally. Energetically, the most beneficial was the arrangement of orthohydrogen atoms in zigzag chains on CNT $(6,6)$ surface extending along the tube axis. In this case, the gain is $1.07 \mathrm{eV}$ compared to free $\mathrm{H}_{2}$. When placing pairs of $\mathrm{H}$ atoms normal to the tube axis, CNT ruptures along the axis thereby forming the saddle-shaped structure in the cross section with the gap between the edges $d_{\mathrm{HH}}$ equal to $2.18 \AA$. The weak interactions between hydrogenated edges of the "saddle" prevents from the obtained sheet straightening inevitable otherwise and turning it into a graphene sheet.

One of the most important impurities to carbon nanomaterials is nitrogen. There are various ways of $\mathrm{N}$ introduction. In [39], 4 types of CNT nitrogenation are considered as follow: direct replacement of carbon atoms by nitrogen atoms, replacement with a vacancy creation, chemosorption of $\mathrm{N}$ atoms and addition of $\mathrm{NH}_{2}$ group. It is found that the replacement of $\mathrm{N}$, chemosorption of $\mathrm{N}$ and $-\mathrm{NH}_{2}$ group strongly modifies electron properties of the semiconducting CNT $(10,0)$. So, substitution by nitrogen results in appearance of new states in the upper half of the band gap, that is, it allows $n$-type doping. The difference between the substitutional doping and the attachment of functional groups to the walls is also in the absence of perturbation 
of $s p^{2}$-hybridization of carbon atom orbitals. At the increased content of embedded $\mathrm{N}$ atoms up to 2.5 at $\%$, a transition of CNT $(10,0)$ to the metallic conductivity type is revealed. At the same time, the replacement by nitrogen with concurrent formation of carbon vacancies even with 7.7 at $\%$ only results in the reduction of $E_{\mathrm{g}} \mathrm{CNT}(10,0)$ to $\sim 0.2 \mathrm{eV}$, that corresponds to characteristics of narrow-gap semiconductors. Doping of metallic CNT $(5,5)$ with nitrogen considerably increases the density of states near the Fermi level and can therefore enhance the reactivity of such CNT. Calculations of ionization potential of nitrided CNT show its reduction to $25 \%$ as compared with pure tubes. The latter is of great importance to improve the CNT emission properties exposed to the external electric field (field emission).

Apart from nitrogen, the researchers also express their interest to introduction of boron atoms to carbon nanomaterials that have the close value of atomic radius with respect to carbon and thus can easily replace the latter. From the technological point of view, co-doping with nitrogen and boron is important to occur directly in process of CNT growing by CVD method [40]. While introduction of nitrogen or boron alone does not change the conductivity type of metallic CNT, introduction both of these two elements induce the appearance of the band gap with the value that can be controlled by varying the concentration of impurity atoms [41, 42].

The works $[43,44]$ investigated CNT stability and electron properties with fluorine atoms added. The geometry of CNT $(5,5)-\mathrm{F}$ complexes is studied and estimated using the SCC-DFTB approximate method where the binding energy of $\mathrm{F}$ atom during chemisorption on the nanotube surface equals to $E_{\mathrm{b}}(\mathrm{F})=5.82 \mathrm{eV}$ at the $\mathrm{C}-\mathrm{F}$ bond length at $1.34 \AA$. It is shown that the pair adsorption is preferable, and the tube walls are exposed to strong deformations that are not observed when using less active impurities (Fig. 7). It should be noted that the task to predict structural forms of fluorinated carbon materials, and thus structure-dependent electronic and optical characteristics is complicated with a wide range of external factors that allow fluorine doping.
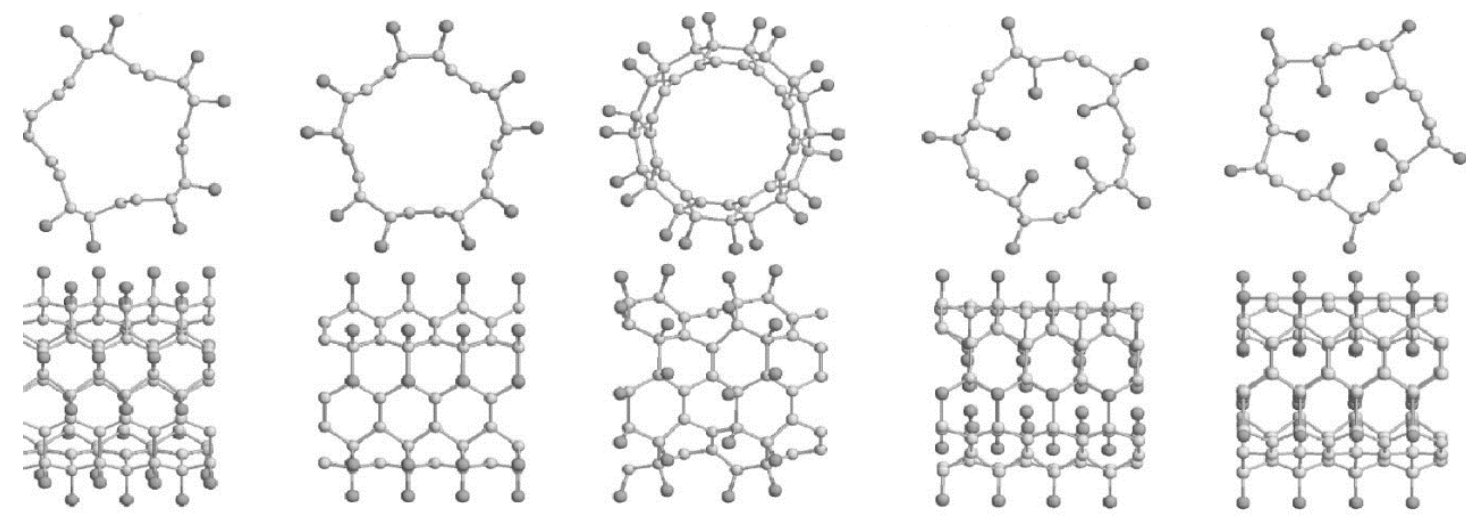

Fig. 7. Optimized SWCNT(5,5)-F structures at various placement of fluorine atoms [43].

Experimental studies show that fluorination of carbon materials is uniform and is not dependent on the fluorine concentration [45]. However, numerical simulation points to the importance of the arrangement of $\mathrm{F}$ atoms on tube walls. Thus, in the case of adsorption of pairs of $\mathrm{F}$ atoms perpendicular to the CNT axis, they are subject to fragmentation.

Similarly with nanotubes, graphene doping with fluorine is considered as one of possible effective ways to modify its properties that helps to control electronic characteristics (primarily by $E_{\mathrm{g}}$ value), as well as the first stage of subsequent chemical processing or doping [46-48]. Using USPP-PW method implemented in the Quantum ESPRESSO package, in [46] the studies of fluorinated layers of graphene for different placements and concentrations of $\mathrm{F}$ atoms have been carried out. It should be noted that the advantage of work [46] is the use of the plane wave basis unlike many other studies of nanocarbon materials. Being a more resourceconsuming in practice, the plane wave basis, due to its completeness, is free of the significant deficiency of localized basis, typical for traditional quantum chemistry, that is, the uncontrolled BSSE error. As per estimations, the bonding energy of $\mathrm{F}$ atoms to the graphene surface is within $E_{\mathrm{b}}(\mathrm{F})=2-2.5 \mathrm{eV}$ and the maximum stability of the doped structure is achieved at $25 \%$ of fluorine coating $\left(\mathrm{C}_{4} \mathrm{~F}\right)$. This theoretical result explains the saturation of samples with fluorine when coated by $25 \%$ as observed in the experiment. Calculations in [46] also indicate that by total bilateral fluorination, the graphene translates to the insulating state with the direct band gap $E_{\mathrm{g}}=3.07 \mathrm{eV}$. Due to the use of PBE-GGA approximation that systematically underestimates the band gap width, the true value of $E_{\mathrm{g}}$ in GR-F system can be expected to be higher. This conclusion is proved by more accurate quasiparticle calculations [47], where $E_{\mathrm{g}}$ of fluorographene was found to be $6.98-8.12 \mathrm{eV}$.

The work [49] focuses on the increased reactivity of carbon nanomaterials with structural defects. The exposure to atmospheric factors will result in the reaction of defective sites with oxygen and CNT oxidization. To investigate possibilities to use CNT as sensitive elements of gas sensors, the authors of [49] performed studies within DFT-LDA of interaction of the oxidized tubes with a range of common molecules: $\mathrm{NO}_{2}, \mathrm{NH}_{3}, \mathrm{CO}, \mathrm{CO}_{2}, \mathrm{H}_{2} \mathrm{O}$. It is revealed that vacancies, resulting from the reaction between $\mathrm{CNT}$ and oxygen, 
cause an increase in the strength of interaction between CNT and external molecules. Chemisorption of test molecules causes quite considerable charge exchange with CNT that changes the tube transport properties and increases the likelihood of foreign molecules detection in the environment. CNT properties are most sensitive to the presence of $\mathrm{NO}_{2}, \mathrm{NH}_{3}$ and $\mathrm{CO}$ molecules, and less sensitive to $\mathrm{CO}_{2}$ molecules.

Unlike sources above, the studies [50-55,57,58] focused on $a b$ initio study of bond formation between external molecular groups and carbon nanomaterials as per non-covalent mechanism. Non-covalent functionalization retains mechanical and electronic properties of CNT and graphene, which is advantageous in certain cases.

The interaction of semiconducting SWCNT $(14,0)$ with molecular groups $-\mathrm{NH}_{2},-\mathrm{CN},-\mathrm{CH}_{3},-\mathrm{CHOCH}_{2}$ by the non-covalent mechanism was studied in [50] by calculations based on DFT local approximation using the plane wave basis and norm-conserving Hamann pseudopotentials. To verify reliability of this calculation method, binding energy $E_{\mathrm{b}}$ of $\mathrm{O}_{2}$ molecule to CNT was also computed by the quantum Monte Carlo method (QMC), that reproduces well the correlation energy and, thus, is highly accurate. The QMC calculation of $E_{\mathrm{b}}$ $(0.06 \mathrm{eV})$ was comparable with LDA result $(0.10 \mathrm{eV})$, whereas the GGA resulted in binding energy even opposite in sign. Based on that, the authors of [50] conclude on the greater reliability of theory within DFT-LDA when describing weak interactions in the considered structures. Performed LDA calculations for the given groups resulted in $E_{\mathrm{b}}$ values within the range of $0.09-0.15 \mathrm{eV}$ and the equilibrium separation between the CNT and molecule is $2.1-2.9 \AA$ in case of neutral CNT. This work pointed to the greater importance of CNT electric charge when forming the non-covalent bond. Thus, the environment of CNT can greatly affect its adhesive properties.

In [51], with using VASP package and various exchange-correlation functionals (LDA, GGA, M06), PP-PW calculations were performed within DFT to determine the structural parameters and bonding energy of the range of aromatic hydrocarbons $\left(\mathrm{C}_{6} \mathrm{H}_{6}, \mathrm{C}_{7} \mathrm{H}_{8} \mathrm{O}\right.$, $\left.\mathrm{C}_{7} \mathrm{H}_{8} \mathrm{~S}, \mathrm{C}_{16} \mathrm{H}_{10}, \mathrm{C}_{17} \mathrm{H}_{12} \mathrm{O}, \mathrm{C}_{17} \mathrm{H}_{12} \mathrm{~S}, \mathrm{C}_{10} \mathrm{H}_{16}, \mathrm{C}_{14} \mathrm{H}_{20}\right)$ that interact with the SWCNT surface. It is established

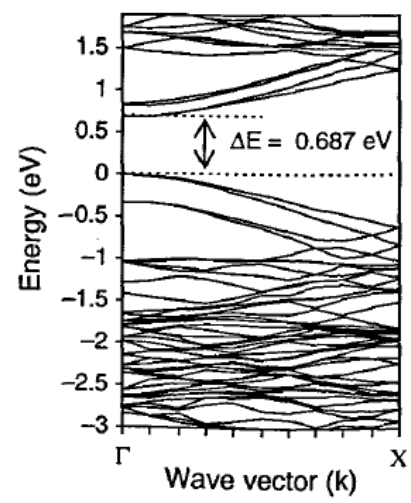

(a) SWCNT

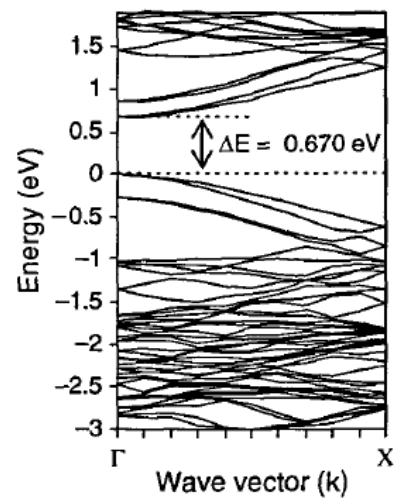

(b) $\mathrm{H}_{2} \mathrm{SO}_{4}$-doped SWCNT that aromatic molecules are stronger bound to CNT as compared with fully saturated non-aromatic molecules. The binding energy is $0.2-0.5 \mathrm{eV}$, where higher values correspond to the molecules of larger size. Aromatic rings are placed above the CNT carbon hexagons like $\mathrm{AB}$ graphite lattice, which helps to conclude on the appropriate type of chemical bond. Even more complex impurities are investigated in the literature, for example, in work [52] the DFT-D method was employed to study CNT interaction with tetraphenylporphyrin molecules. Including the dispersion interactions improved the accuracy to model the structural properties of the complex, and showed its high stability, which is important for application in the electrocatalysis.

Since the acid treatment is one of the standard purification method of nanocarbon materials, the modeling and analysis of interaction between them and nitric and sulfuric acids is of high practical value. The adsorption of sulfuric acid molecules on CNT surface was studied in [53]. The semiconducting CNT $(12,4)$ was used as the source object for modeling. Calculations of the binding energy $E_{\mathrm{b}}$ with $\mathrm{PBE}$ functional resulted in the value $E_{\mathrm{b}}\left(\mathrm{H}_{2} \mathrm{SO}_{4}\right)$ of $0.20 \mathrm{eV}$ to be compared with interaction energies of CNT and such molecules as $\mathrm{O}_{2}$ and $\mathrm{Br}_{2}(0.19$ and $0.25 \mathrm{eV}$, accordingly). Due to small interaction energy, the tube is not exposed to considerable deformation to be normally observed during covalent functionalization. The authors defined the equilibrium distance from the $\mathrm{H}$ atom of $\mathrm{H}_{2} \mathrm{SO}_{4}$ molecule to the nearest $\mathrm{C}$ atom of the nanotube, $2.38 \AA$, and estimated the charge transmitted by CNT to impurity molecules as 0.008 . The comparison of electron spectra and density of electronic states of CNT prior and after doping shows their almost complete identity (Fig. 8). The detailed analysis reveals that the $E_{\mathrm{g}}$ value decreases that is connected in [53] with some changes in CNT geometry due to charge transfer. The reducing of the band gap width by doping with $\mathrm{H}_{2} \mathrm{SO}_{4}$ may be one of reasons for the increasing conductivity of carbon nanomaterials when treated with sulfuric acid. Nevertheless, the results of [52] require verification using CNT with the other set of chirality indices, as well as using more complex calculation methods that may provide more reliable data on values of interband transitions.

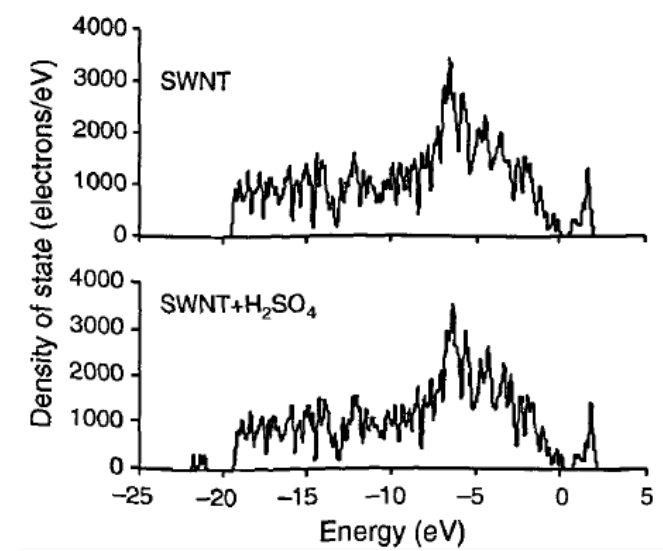

Fig. 8. Electron spectra and density of states of pure $\operatorname{SWCNT}(12,4)$ and the complex $\operatorname{SWCNT}(12,4)-\mathrm{H}_{2} \mathrm{SO}_{4}[53]$. 
The emergence of the new family of exchangecorrelation functionals that can treat dispersion interactions within DFT caused the growth of theoretical research of systems based on graphene layers, where the van der Waals forces play an important role. In $[54,55]$, the estimations of the productivity of various approximations to calculate the exchangecorrelation energy when describing structural and energy characteristics of graphene layers, adsorbed on the (111) surface of $\mathrm{Ni}, \mathrm{Cu}, \mathrm{Pd}, \mathrm{Ag}, \mathrm{Au}$, Pt metals have been done. From practical point of view, understanding of interactions between graphene and metal is of apparent importance to develop next-generation nanoelectronic devices, as well as to select the appropriate substrate

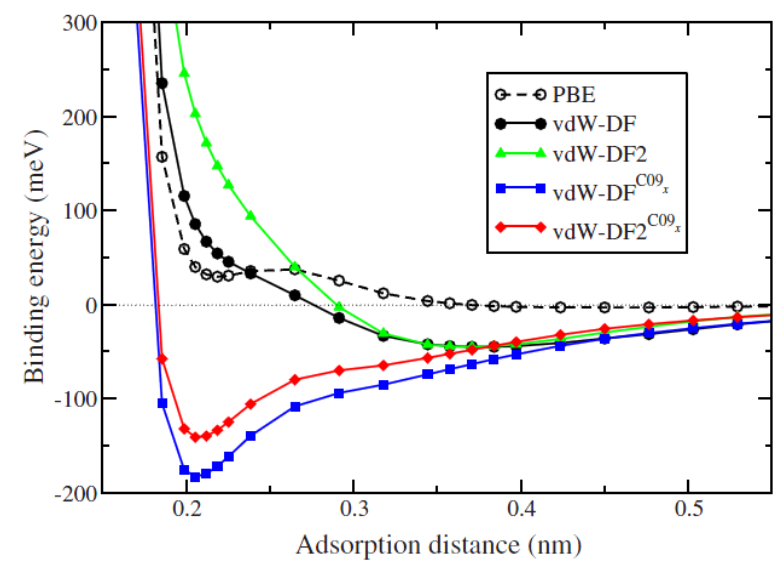

for graphene growth. Studies indicate that a range of metals (Pd(111), $\mathrm{Co}(0001), \mathrm{Ni}(111))$ apparently impact the electron properties of graphene whereas the absorption on substrates (111) $\mathrm{Cu}, \mathrm{Au}, \mathrm{Pt}, \mathrm{Ir}, \mathrm{Ag}$ hardly affects its electron structure. These results are difficult to explain using standard LDA and GGA functionals that well describe the ionic-covalent and metallic bonds, but show large discrepancies against experimental data when modeling systems with significant contribution of the dispersion interactions. The efficiency of various functionals is demonstrated in Fig. 9 where curves of the binding energy are shown in dependence on the distance between the metal substrate and the graphene monolayer.

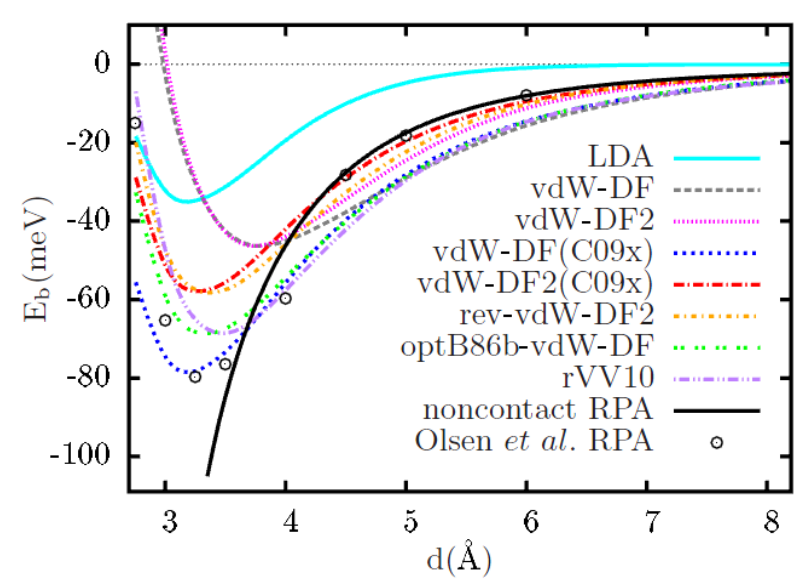

Fig. 9. The bonding energy of graphene with surfaces $\mathrm{Ni}(111)$ [53] (left) and $\mathrm{Ag}(111)$ [54] (right) in terms of one carbon atom.

It is worth noting that $\mathrm{Ag}$ (111) surface is of particular interest to evaluate theoretical results, since it provides the lowest binding energy to the graphene. Fig. 9 shows that different approximations strongly differ from others. The largest error is reported with $\mathrm{PBE}$ functional that predicts instability of GR-Ni(111) system contrary to experimental data. At the same time, new vdW-DF(C09x) and rev-vdW-DF2 functionals show the good level of accuracy when reproducing equilibrium distances, as well as when describing contributions to interaction energy. The last conclusion can be made by comparison with resource-consuming calculation within the random phase approximation (ACFDT-RPA) that allows to calculate the correlation energy of many-electron systems at high accuracy, including systems with van der Waals bonds. Calculations with vdW-DF2(C09x) were also able to describe the appearance of the band gap in the electronic spectrum of graphene on the nickel substrate in good consistency with experimental data [56].

Analysis of calculation results using various vdW-DF functionals to model adsorption of nucleic acid nitrogen bases on the graphene surface (adenine $(A)$, guanine $(\mathrm{G})$, cytosine $(\mathrm{C})$, thymine $(\mathrm{T})$ and uracil (U)) was also performed in $[57,58]$. The values $E_{\mathrm{b}}$ calculated by DFT are in the sequence of $\mathrm{G}>\mathrm{A}>\mathrm{T}$ $>\mathrm{C}>\mathrm{U}$ (either $\mathrm{G}>\mathrm{A}>\mathrm{T} \sim \mathrm{C}>\mathrm{U}$ [57]), which is consistent with the quantum chemical calculations using the Möller-Plesset perturbation theory of 2nd order.
$\mathrm{Ab}$ initio calculations made with clearly defined parameters of the object being studied, appear the good tool to study the effect of functionalization on the conductivity of nanosized systems as one of the most important characteristics of the material to be used in electronic devices. Such studies were carried out for CNT in the range of works [59-67] using the formalism of non-equilibrium Green's functions with the numerical implementation of the method in TranSIESTA program (except [59]) that is based on SIESTA package implementing DFT with the localized atomic basis. In [59], the approach to the electron structure calculations based on the use of maximally localized Wannier functions (MLWFs) as the basis has been employed. The authors used nitrophenyl and aminophenyl as ligands. Calculations showed that the covalently bound groups act as strong scattering centers, reducing the CNT ballistic conduction by tens of percent $(42 \%)$ already at low degrees of functionalization. Transport properties of CNT with attached fullerenes (the so-called nanobads) were studied in [60]. CNT relaxation was performed by using Brenner empirical potentials realized in the Gulp package. It is concluded from results obtained that the CNT $(8,8)$ conductivity with fullerenes decreases within the range of energies higher than the Fermi energy $E_{\mathrm{F}}$ as compared with the ideal nanotube. For energies smaller than $E_{\mathrm{F}}$ the transmission spectrum has the plato-like area and is close to that of the initial nonfunctionalized CNT. The authors note the great impact 
on the transmission spectrum of the "neck" region connecting the fullerene with CNT.

As seen from the range of experimental studies, the electrical resistance of CNT films can be reduced by adding metallic nanoparticles. Therefore, the CNT conduction with gold nanoparticles placed between CNT ends was studied in [61]. The obtained results showed that if the Au cluster contains the odd number of atoms (13), the conduction considerably increases and becomes higher for an order as compared with the transitions between the CNT without additives, whereas the opposite is true in case of the even number of Au (14) atoms. This effect is due to that Au clusters with the even number of particles have a non-zero gap HOMO-LUMO due to completeness of the electronic shell. Simulation of graphene nanoribbons with zigzag boundaries [62] also suggests the possibility to control their electrical properties by doping with metal atoms $(\mathrm{Cu})$. A challenging result may be obtained by the mutual combination of vacancies and adatoms. For example, in [63] the properties of defective CNT with randomly placed nitrogen atoms and vacancies along the tube were studied. The substitution of carbon by the nitrogen results in suppression of one of the conducting spin channels, while the conductivity in the second channel remains almost unchanged. Based on calculation of total CNT energy it was determined that the most stable defect in nitrogen doped CNT is the carbon divacancy surrounded by nitrogen atoms similar to the pyridine molecule. These regions interact with ammonia molecules causing their dissociation and binding of $-\mathrm{NH}_{2}$ complex which may be successfully used when designing relevant sensor devices.

Studies in [64] explained in theory the experimental dependence of the conductivity of CNT meshes exposed to oxygen and nitrogen gases. Simulation of
CNT intersections with oxygen and nitrogen molecules placed between tubes show that the conduction increases due to proximity of the $\mathrm{O}_{2}$ HOMO to the CNT Fermi level, causing an increase in the probability of electron tunneling. The similar effect though a bit weaker occurs in case of nitrogen molecules. The work [65] analyzed the graphene sheet and $\operatorname{CNT}(10,10)$, containing the transition metal atom - Fe or $\mathrm{V}$ over one of central carbon cells. It was found out that the presence of metal adatom result in the spinselective conduction at certain energies. The mechanism of this effect relates to Fano antiresonance. CNT $(5,5)$ properties were analyzed in [66] containing mono-, diand trivacancies of carbon that correspond to magnetic impurities in their properties. The outcome of works [66] and [65] prove the conductance sensitivity to the spin in some energy windows. Thus, according to [66] in case of the monovacancy, the conduction at the Fermi level significantly drops for one spin channel (spin up), while it remains almost unchanged for the other.

\section{CONCLUSION}

Thus, researches reviewed show the importance of modern computational methods to analyze structural, electronic and energetic characteristics of carbon nanomaterials. When modeling defective or doped systems, it is possible to predict their key physical and chemical properties and assess opportunities of their application in technology. Improvement of the exchangecorrelation functionals with the possibility to treat the dispersion effects widens the scope of the DFT tool application with systems with significant non-covalent interactions.

The work is performed as a part of the government assignment by the Ministry of Education and Science of the Russian Federation (Project № 3.392.2014K).

\section{REFERENCES}

1. Burke K. Perspective on density functional theory. J. Chem. Phys., 2012, vol. 136, pp. 150901-1-150901-9.

2. Scuseria G.E., Staroverov V.N. Progress in the development of exchange-correlation functionals. Chapter 24. Theory and applications of computational chemistry: the first 40 years (a volume of technical and historical perspectives). Amsterdam: Elsevier, 2005, pp. 669-724.

3. Klimeš J., Michaelides A. Perspective: Advances and challenges in treating van der Waals dispersion forces in density functional theory. J. Chem. Phys., 2012, vol. 137, pp. 120901-1-120901-12.

4. Grimme S. Semiempirical GGA-type density functional constructed with a long-range dispersion correction. J. Comput. Chem., 2006, vol. 27, pp. 1787-1799.

5. Tkatchenko A., Scheffler M. Accurate molecular van der Waals interactions from ground-state electron density and free-atom reference data. Phys. Rev. Lett., 2009, vol. 102, pp. 073005-1-073005-4.

6. Dion M., Rydberg H., Schröder E., Langreth D.C., Lundqvist B.I. van der Waals density functional for general geometries. Phys. Rev. Lett., 2004, vol. 92, pp. 246401-1-246401-4.

7. Lee K., Murray E.D., Kong L., Lundqvist B.I., Langreth D.C. Higher-accuracy van der Waals density functional. Phys. Rev. B, 2010, vol. 82, pp. 081101-1-081101-4.

8. Cooper V.R. Van der Waals density functional: an appropriate exchange functional. Phys. Rev. B, 2010, vol. 81, pp. 161104-1161104-4.

9. Hamada I. van der Waals density functional made accurate. Phys. Rev. B, 2014, vol. 89, pp. 121103-1-121103-4.

10. Sarkisov S.Y., Kosobutsky A.V., Shandakov S.D. Effect of van der Waals interactions on the structural and binding properties of GaSe. J. Sol. State Chem., 2015, vol. 232, pp. 67-72.

11. Brudnyi V.N., Sarkisov S.Yu., Kosobutsky A.V. Electronic properties of GaSe, InSe, GaS and GaTe layered semiconductors: charge neutrality level and interface barrier heights. Semicond. Sci. Technol., 2015, vol. 30, no. 11, pp. 115019-1-115019-9.

12. Akdim B., Kar T., Duan X., Pachter R. Functionalization of single-wall carbon nanotubes: an assessment of computational methods. Proceedings of 4th International Conference "Computational Science - ICCS 2004”. Krakow, Poland, 2004, pp. $260-267$.

13. Ramanathan T., Fischer F.T., Ruo R.S., Brinson L.C. Amino-functionalized carbon nanotubes for binding to polymers and biological systems. Chem. Mater., 2005, vol. 17, pp. 1290-1295. 
14. Pan H., Feng P., Lin J.Y. Ab initio study of OH-functionalized single-wall carbon nanotubes. Phys. Rev. B, 2004, vol. 70, pp. 245425-1-245425-5.

15. Chelmecka E., Pasterny K., Kupka T., Stobinski L. DFT studies of OH-functionalized open-ended zigzag, armchair, and chiral single wall carbon nanotubes. Phys. Status Solidi A, 2011, vol. 208, no. 8, pp. 1774-1777.

16. Veloso M.V., Souza Filho A.G., Mendes Filho J., Fagan S.B., Mota R. Ab initio study of covalently functionalized carbon nanotubes. Chem. Phys. Lett., 2006, vol. 430, pp. 71-74.

17. Doudou B.B., Chen J., Vivet A., Poîlane C., Ayachi M. Size-dependent properties of amino-functionalized single walled carbon nanotubes. Comput. Theor. Chem., 2011, vol. 967, pp. 231-234.

18. Wang C., Zhou G., Liu H., Wu J., Qiu Y., Gu B.-L., Duan W. Chemical functionalization of carbon nanotubes by carboxyl groups on Stone-Wales defects: a density functional theory study. J. Phys. Chem. B, 2006, vol. 110, pp. 10266-10271.

19. Doudou B.B., Chen J., Vivet A., Poîlane C., Ayachi M. Role of Stone-Wales defects on the functionalization of $(8,0)$ single wall carbon nanotubes by the amine group: ab initio study. Physica E, 2011, vol. 44, pp. 120-123.

20. Xu Y.-J., Li J.-Q. The interaction of $\mathrm{N}_{2}$ with active sites of a single-wall carbon nanotube. Chem. Phys. Lett., 2005, vol. 412, no. 4-6, pp. 439-443.

21. Basiuk V.A. ONIOM studies of chemical reactions on carbon nanotube tips: effects of the lower theoretical level and mutual orientation of the reactants. J. Phys. Chem. B, 2003, vol. 107, no. 34, pp. 8890-8897.

22. Sofo J.O., Chaudhari A.S., Barber G.D. Graphane: a two-dimensional hydrocarbon. Phys. Rev. B, 2007, vol. 75, pp. 153401-1-153401-4.

23. Ellias D.C., Nair R.R., Mohiuddin T.G.M., Morozov S.V., Blake P., Halsall M.P., Ferrari A.C., Boukhvalov D.W., Katsnelson M.I., Geim A.K., Novoselov K.S. Control of graphene's properties by reversible hydrogenation: evidence for graphane. Science, 2009, vol. 323, no. 5914, pp. 610-613.

24. Pedersen T.G., Flindt C., Pedersen J., Mortensen N.A., Jauho A.pp. Graphene antidot lattices: designed defects and spin qubits. Phys. Rev. Lett., 2008, vol. 100, pp. 136804-1-136804-4.

25. Appelhans D.J., Carr L.D., Lusk M.T. Embedded ribbons of graphene allotropes: an extended defect perspective. New J. Phys., 2010, vol. 12, pp. 125006-1-125006-21.

26. Peng X., Ahuja R. Symmetry breaking induced bandgap in epitaxial graphene layers on SiC. Nano Lett., 2008, vol. 8, no. 12, pp. 4464-4468.

27. Lahiri J., Lin Y., Bozkurt P., Oleynik I.I., Batzill M. An extended defect in graphene as a metallic wire. Nat. Nanotechnol., 2010, vol. 5, pp. 326-329.

28. Banhart F., Kotakoski J., Krasheninnikov A.V. Structural defects in graphene. ACSnano, 2011, vol. 5, no. 1, pp. $26-41$.

29. Lu Y.H., Feng Y.P. Band-gap engineering with hybrid graphane-graphene nanoribbons. J. Phys. Chem. C, 2009, vol. 113, pp. 20841-20844.

30. Boukhvalov D.W., Katsnelson M.I., Lichtenstein A.I. Hydrogen on graphene: electronic structure, total energy, structural distortions and magnetism from first-principles calculations. Phys. Rev. B, 2008, vol. 77, pp. 035427-1-035427-7.

31. Boukhvalov D. W. Repair of magnetism in oxidized graphene nanoribbons. Chem. Phys. Lett., 2011, vol. 501, pp. $396-399$.

32. Milowska K., Birowska M., Majewski J.A. Structural and electronic properties of functionalized graphene. Acta Phys. Pol. A, 2011, vol. 120, no. 5, pp. 842-844.

33. Chang K., Berber S., Tomanek D. Transforming carbon nanotubes by silylation: an ab initio study. Phys. Rev. Lett., 2008, vol. 100, pp. 236102-1-236102-4.

34. Curran S.A., Cech J., Zhang D., Dewald J.L., Avadhanula A., Kandadai M., Roth S.J. Thiolation of carbon nanotubes and sidewall functionalization. Mater. Res., 2006, vol. 21, pp. 1012-1018.

35. Nakamura T., Ohana T., Ishihara M., Hasegawa M., Koga Y. Chemical modification of single-walled carbon nanotubes with sulfur-containing functionalities. Diamond Relat. Mater., 2007, vol. 16, pp. 1091-1094.

36. Denis A.P. Theoretical Investigation of the stability, electronic and magnetic properties of thiolated single-wall carbon nanotubes. Int. J. Quantum Chem., 2009, vol. 109, pp. 772-781.

37. Cho E., Shin S., Yoon Y.-G. First-principles studies on carbon nanotubes functionalized with azomethine ylides. J. Phys. Chem. C, 2008, vol. 112, pp. 11667-11672.

38. Miller G.P., Kintigh J., Kim E., Weck P.F., Berber S., Tomanek D. Hydrogenation of single--wall carbon nanotubes using polyamine reagents: combined experimental and theoretical study. J. Am. Chem. Soc., 2008, vol. 130, no. 7, pp. $2296-2303$.

39. Lim S.H., Li R., Ji W., Lin J. Effects of nitrogenation on single-walled carbon nanotubes within density functional theory. Phys. Rev. B, 2007, vol. 76, pp. 195406-1-195406-16.

40. Wang W.L., Bai X.D., Liu K.H., Xu Z., Golberg D., Bando Y., Wang E.G. Direct synthesis of B-C-N single-walled nanotubes by bias-assisted hot filament chemical vapor deposition. J. Am. Chem. Soc., 2006, vol. 128, pp. 6530-6531.

41. Chen L.-N., Ma S.-S., OuYang F.-P., Xiao J., Xu H. First-principles study of metallic carbon nanotubes with boron/nitrogen co-doping. Chin. Phys. B, 2011, vol. 20, no. 1, pp. 017103-1-017103-7.

42. Xu Z., Lu W., Wang W., Gu C., Liu K., Bai X., Wang E., Dai H. Converting metallic single-walled carbon nanotubes into semiconductors by boron/nitrogen co-doping. Adv. Mater, 2008, vol. 20, pp. 3615-3619.

43. Park K.A., Choi Y.S., Lee Y.H., Kim C. Atomic and electronic structures of fluorinated single-walled carbon nanotubes. Phys. Rev. B, 2003, vol. 68, pp. 045429-1-045429-8.

44. Margulis Vl.A., Muryumin E.E. Chemisorption of single fluorine atoms on the surface of zigzag single-walled carbon nanotubes: a model calculation. Physica B, 2007, vol. 390, pp. 134-142.

45. Brzhezinskaya M.M., Muradyan V.E., Vinogradov N.A., Preobrajenski A.B., Gudat W., Vinogradov A.S. Electronic structure of fluorinated multiwalled carbon nanotubes studied using $\mathrm{x}$-ray absorption and photoelectron spectroscopy. Phys. Rev. B, 2009, vol. 79, pp. 155439-1-155439-12.

46. Robinson J.T., Burgess J.S., Junkermeier C.E., Badescu S.C., Reinecke T.L., Perkins F.K., Zalalutdniov M.K., Baldwin J.W., Culbertson J.C., Sheehan P.E. Properties of fluorinated graphene films. Nano Lett., 2010, vol. 10, pp. 3001-3005.

47. Karlicky F., Otyepka M. Band gaps and optical spectra of chlorographene, fluorographene and graphane from $\mathrm{G}_{0} \mathrm{~W}_{0}, \mathrm{GW}_{0}$ and GW calculations on top of PBE. J. Chem. Theory Comput., 2013, vol. 9, pp. 4155-4164. 
48. Zhou J., Wu M.M., Zhou X., Sun Q. Tuning electronic and magnetic properties of graphene by surface modification. Appl. Phys. Lett., 2009, vol. 95, pp. 103108-1-103108-3.

49. Zanolli Z., Charlier J.-C. Defective carbon nanotubes for single-molecule sensing. Phys. Rev. B, 2009, vol. 80, pp. 155447-1-155447-6.

50. Cicero G., Grossmann J.C., Galli G. Adhesion of single functional groups to individual carbon nanotubes: electronic effects probed by ab initio calculations. Phys. Rev. B, 2006, vol. 74, pp. 035425-1-035425-5.

51. Lim S., Park N. Ab initio study of noncovalent sidewall functionalization of carbon nanotubes. Appl. Phys. Lett., 2009, vol. 95, pp. 243110-1-243110-4.

52. Orellana W. Single- and double-wall carbon nanotubes fully covered with tetraphenylporphyrins: stability and optoelectronic properties from ab initio calculations. Chem. Phys. Lett., 2015, vol. 634, pp. 47-52.

53. Choi J.I., Jang S.S. Structural and electronic properties of sulfuric acid-doped single-walled carbon nanotubes. J. Comput. Theor. Nanosci., 2010, vol. 7, pp. 232-236.

54. Hamada I., Otani M. Comparative van der Waals density-functional study of graphene on metal surfaces. Phys. Rev. B, 2010, vol. 82, pp. 153412-1-153412-4.

55. Loncaric I., Despoja V. Benchmarking van der Waals functionals with noncontact RPA calculations on graphene- $\mathrm{Ag}(111)$. Phys. Rev. B, 2014, vol. 90, pp. 075414-1-075414-6.

56. Grüneis A., Vyalikh D.V. Tunable hybridization between electronic states of graphene and a metal surface. Phys. Rev. B, 2008, vol. 77, pp. 193401-1-193401-4.

57. Le D., Kara A., Schroder E., Hyldgaard P., Rahman T.S. Physisorption of nucleobases on graphene: a comparative van der Waals study. J. Phys.: Condens. Matter., 2012, vol. 24, pp. 424210-1-424210-7.

58. Lee J.-H., Choi Y.-K., Kim H.-J., Scheicher R.H., Cho J.-H. Physisorption of DNA nucleobases on h-BN and graphene: vdWcorrected DFT calculations. J. Phys. Chem. C, 2013, vol. 117, no. 26, pp. 13435-13441.

59. Lee Y.-S., Nardelli M.B., Marzari N. Band structure and quantum conductance of nanostructures from maximally localized Wannier functions: the case of functionalized carbon nanotubes. Phys. Rev. Lett., 2005, vol. 95, pp. 076804-1-076804-4.

60. Furst J.A., Hashemi J., Markussen T., Brandbyge M., Jauho A.P., Nieminen R.M. Electronic transport properties of fullerene functionalized carbon nanotubes: ab initio and tight-binding calculations. Phys. Rev. B, 2009, vol. 80, pp. 035427-1-035427-4.

61. Khoo K.H., Chelikowsky J.R. Electron transport across carbon nanotube junctions decorated with Au nanoparticles: density functional calculations. Phys. Rev. B, 2009, vol. 79, pp. 205422-1-205422-6.

62. Berahman M., Sheikhi M.H. Transport properties of zigzag graphene nanoribbon decorated with copper clusters. J. Appl. Phys., 2014, vol. 116, pp. 093701-1-093701-8.

63. Rocha A.R., Rossi M., da Silva A.J.R., Fazzio A. Realistic calculations of carbon-based disordered systems. J. Phys. D: Appl. Phys., 2010, vol. 43, pp. 374002-1-374002-12.

64. Mowbray D.J., Morgan C., Thygesen K.S. Influence of $\mathrm{O}_{2}$ and $\mathrm{N}_{2}$ on the conductivity of carbon nanotube networks. Phys. Rev. B, 2009, vol. 79, pp. 195431-1-195431-6.

65. Furst G.A., Brandbyge M., Jauho A.-P., Stokbro K. Ab initio study of spin-dependent transport in carbon nanotubes with iron and vanadium adatoms. Phys. Rev. B, 2008, vol. 78, pp. 195405-1-195405-7.

66. Zanolli Z, Charlier J.-C. Spin transport in carbon nanotubes with magnetic vacancy-defects. Phys. Rev. B, 2010, vol. 81, pp. 165406-1-165406-6.

Alexey V. Kosobutsky

Cand.Sci.(Phys.-Math.), Senior Fellow for the Kemerovo State University, Kemerovo, Russian Federation

\section{Sergey D. Shandakov}

Dr.Sci.(Phys.-Math.), Professor, Head of Chair for Experimental Physics of Kemerovo State University, Kemerovo, Russian Federation

\section{Albert G. Nasibulin}

Dr.Sci.(Eng.), Professor, Skolkovo Institute of Science and Technology, Moscow, Russian Federation 